\title{
HS1 protein is differentially expressed in chronic lymphocytic leukemia patient subsets with good or poor prognoses
}

\author{
Cristina Scielzo, ${ }^{1}$ Paolo Ghia, ${ }^{1}$ Antonio Conti, ${ }^{2}$ Angela Bachi, ${ }^{3}$ Giuseppe Guida, ${ }^{4}$ Massimo Geuna, ${ }^{5}$ \\ Massimo Alessio, ${ }^{2}$ and Federico Caligaris-Cappio'
}

\begin{abstract}
${ }^{1}$ Department of Oncology, Università Vita e Salute-San Raffaele, Milan, Italy. ${ }^{2}$ Proteome Biochemistry Unit and ${ }^{3}$ Mass Spectrometry Unit, San Raffaele Scientific Institute, Milan, Italy. ${ }^{4}$ Department of Oncological Sciences, Università di Torino, Candiolo, Italy. ${ }^{5}$ Laboratory of Cancer Immunology, Institute for Cancer Research and Treatment, Candiolo, Italy.
\end{abstract}

\begin{abstract}
We used a proteomic approach for identifying molecules involved in the pathogenesis of chronic lymphocytic leukemia (CLL). We investigated 14 patients who were completely concordant for $I g V_{H}$ mutational status (unmutated vs. mutated), CD38 expression (positive vs. negative), and clinical behavior (progressive vs. stable); these patients were characterized as having either poor or good prognoses. The 2 patient subsets differed in the expression of hematopoietic lineage cell-specific protein 1 (HS1). In patients with poor prognoses, most HS1 protein was constitutively phosphorylated, whereas only a fraction was phosphorylated in patients with good prognoses. This difference was investigated in a larger cohort of 26 unselected patients. The survival curve of all 40 patients analyzed revealed that patients with predominately phosphorylated HS1 experience a significantly shorter median survival time. As HS1 is a protein pivotal in the signal cascade triggered by $B$ cell receptor (BCR) stimulation, we studied its pattern of expression following BCR engagement. Normal mature B cells stimulated by anti-IgM shifted the non- or less-phosphorylated form of HS1 toward the more phosphorylated form. Naive B cells showed both HS1 forms while memory B cells expressed mainly the phosphorylated fraction. These data indicate a central role for antigen stimulation in CLL and suggest a new therapeutic target for patients with aggressive disease.
\end{abstract}

\section{Introduction}

Chronic lymphocytic leukemia (CLL), the most common B cell malignancy in adults, is characterized by the relentless expansion of monoclonal mature B lymphocytes. The biased expression of certain immunoglobulin variable beavy chain $\left(\operatorname{Ig} V_{H}\right)$ genes $(1-5)$, the existence of similarities among Ig rearrangements (5-8), and the presence of $\operatorname{Ig} V_{H}$ somatic mutations together with the typical expression of gene products associated with B cell signaling and activation (9) raise the possibility that an antigenic drive may be instrumental in malignant cell growth. The analysis of $\operatorname{Ig} V_{H}$ somatic mutations, which track the clonal history to an in vivo activation of B cell receptor-mediated (BCR-mediated) activation, differentiates 2 distinct CLL subsets, 1 with somatically mutated and 1 with unmutated $\operatorname{Ig} V_{H}$ genes $(1,4)$. The 2 subsets have a markedly different prognosis, with unmutated CLL (U-CLL) patients presenting a considerably shorter survival period $(2,10)$.

The presence of $\operatorname{Ig} V_{H}$ somatic mutations suggests a role for BCR activation in the natural history of mutated CLL (M-CLL), though these cases are typically unresponsive to BCR stimulation in vitro $(11,12)$ and thus resemble $B$ cells that have undergone receptor desensitization following chronic stimulation by antigen

Nonstandard abbreviations used: BCR, B cell receptor; CLL, chronic lymphocytic leukemia; 2-DE, 2D electrophoresis; HS1, hematopoietic lineage cell-specific protein 1; $\mathrm{IgV}_{H}$, immunoglobulin variable heavy chain; MALDI-TOF, matrix-assisted laser desorption/ionization-time-of-flight; M-CLL, mutated CLL; Mr, relative molecular mass; MS, mass spectrometry; $\mathrm{PI}$, isoelectric point; $\lambda$ PPase, $\lambda$ protein phosphatase; U-CLL, unmutated CLL; ZAP-70, $\zeta$ chain-associated protein of $70 \mathrm{kDa}$. Conflict of interest: The authors have declared that no conflict of interest exists.
(13). Conversely, though expressing germline $\operatorname{Ig} V_{H}$ genes, U-CLL strongly responds in vitro to anti-IgM stimulation, as shown by an increase in global tyrosine phosphorylation $(11,12)$, which suggests that $\mathrm{U}$-CLL carry a more competent $\mathrm{BCR}$, able to receive signals for maintenance or proliferation. In addition, CD38, a signaling molecule that may influence the outcome of BCR signaling once a specific surface expression threshold is reached (e.g., in the presence of IL-2) (14), also tends to be better represented in U-CLL (10). CD38 too has a significant prognostic value, which is not related to the presence or absence of $\operatorname{Ig} V_{H}$ somatic mutations (15). Interestingly, a strong correlation between CD38 expression and responsiveness to signaling via surface $\operatorname{IgM}(16,17)$ has been reported and appears to be enhanced by the expression of the signaling molecule $\zeta$ chain-associated protein of $70 \mathrm{kDa}$ (ZAP-70) $(11,18)$. The expression of ZAP-70 (19), a kinase that shares functions with the spleen tyrosine kinase Syk, is also associated with unmutated $\operatorname{Ig} V_{H}$ status (20-23).

The markedly different response to the stimulation of BCR found in leukemic cells from CLL subsets characterized by a distinct clinical behavior leads to the questions of whether the stimulation of BCR has a role in the onset only or is also involved in the maintenance of the disease and to what extent these 2 possible scenarios may apply to the different subsets of patients. We have used a 2D electrophoresis (2-DE) proteomic approach to study the cells from 40 CLL patients, characterized on the basis of their biological and clinical features. By 2-DE and mass spectrometry (MS) analysis, we have found that hematopoietic lineage cell-specific protein 1 (HS1), an intracellular protein that is pivotal in the signal cascade triggered by BCR stimulation, is differentially expressed and shows distinct features in different subsets of patients. 
Table 1

Clinical and biological features of the CLL patients

\begin{tabular}{|c|c|c|c|c|c|c|c|c|c|c|c|c|}
\hline $\begin{array}{l}\text { Patient } \\
\text { no. }\end{array}$ & $\begin{array}{l}\text { Age } \\
\text { (yr) }\end{array}$ & Sex & $\mathrm{HC}$ & LC & $\begin{array}{c}\text { Clinical } \\
\text { course }\end{array}$ & $\begin{array}{c}\lg V_{H}{ }^{A} \\
(\%)\end{array}$ & $\begin{array}{c}\text { CD38 } \\
(\%)\end{array}$ & $\begin{array}{c}\text { ZAP-70 } \\
\text { expression }\end{array}$ & Stage $^{B}$ & $\begin{array}{c}\text { Follow-up } \\
(\mathrm{mo})\end{array}$ & DeathC & $\begin{array}{l}\text { HS1 protein } \\
\text { spots }^{\mathrm{D}}\end{array}$ \\
\hline 1 & 79 & M & $\lg M / D$ & $\kappa$ & Progressive & 100.0 & 41 & Pos & $0 / A$ & 97 & yes & 1 \\
\hline 2 & 69 & $M$ & $\lg M / D$ & $\lambda$ & Progressive & 100.0 & 81 & Pos & ND & 36 & no & 2 \\
\hline 3 & 53 & M & $\lg M / D$ & $\kappa$ & Progressive & 100.0 & 89 & Pos & 2/B & 48 & yes & 1 \\
\hline 4 & 66 & M & $\lg M / D$ & $\kappa$ & Progressive & 100.0 & 99 & Pos & $4 / C$ & 107 & no & 1 \\
\hline 5 & 82 & M & $\lg M / D$ & $\lambda$ & Progressive & 99.0 & 60 & Pos & 2/B & 162 & no & 1 \\
\hline 6 & 80 & $\mathrm{~F}$ & $\lg M / D$ & $\lambda$ & Progressive & 98.6 & 30 & Pos & 2/B & 84 & yes & 1 \\
\hline 7 & 79 & M & $\lg M / D$ & $\kappa$ & Progressive & 98.6 & 32 & Pos & 2/B & 82 & no & 2 \\
\hline 8 & 53 & M & $\lg M / D$ & $\kappa$ & Stable & 96.2 & 0.1 & Neg & $1 / B$ & 159 & no & 2 \\
\hline 9 & 55 & M & $\lg M / D$ & $\lambda$ & Stable & 95.3 & 0.3 & Neg & $0 / A$ & 107 & no & 1 \\
\hline 10 & 50 & M & $\lg M / D$ & $\kappa$ & Stable & 93.3 & 1.5 & Neg & $0 / A$ & 105 & no & 2 \\
\hline 11 & 71 & $\mathrm{~F}$ & $\lg M / D$ & $\kappa$ & Stable & 92.9 & 3.0 & Neg & $0 / A$ & 199 & no & 2 \\
\hline 12 & 67 & $\mathrm{~F}$ & $\lg M / D$ & $\lambda$ & Stable & 91.8 & 0.2 & Neg & $0 / A$ & 143 & no & 2 \\
\hline 13 & 78 & $\mathrm{~F}$ & $\lg M / D$ & $\kappa$ & Stable & 89.5 & 0.4 & Neg & $0 / A$ & 101 & no & 2 \\
\hline 14 & 72 & $\mathrm{~F}$ & $\lg M / D$ & $\lambda$ & Stable & 88.4 & 0.1 & Neg & $0 / A$ & 53 & no & 2 \\
\hline 15 & 63 & M & $\lg M / D$ & $\kappa$ & Progressive & 100.0 & 93 & Neg & $0 / A$ & 98 & yes & 1 \\
\hline 16 & 73 & $M$ & $\lg M / D$ & $\kappa$ & Progressive & 100.0 & 78 & Neg & $0 / A$ & 117 & no & 1 \\
\hline 17 & 85 & $\mathrm{~F}$ & $\lg M / D$ & $\lambda$ & Progressive & 99.3 & 0.5 & Neg & $1 / A$ & 44 & no & 1 \\
\hline 18 & 76 & $\mathrm{~F}$ & $\lg M / D$ & $\kappa$ & Progressive & 99.0 & 38 & Pos & $0 / A$ & 59 & no & 2 \\
\hline 19 & 76 & $M$ & $\lg M / D$ & $\kappa$ & Progressive & 99.0 & 74 & Pos & $0 / A$ & 101 & no & 2 \\
\hline 20 & 76 & $\mathrm{~F}$ & $\lg M / D$ & $\kappa$ & Progressive & 98.6 & 100. & Pos & 2/B & 13 & no & 1 \\
\hline 21 & 57 & $\mathrm{~F}$ & $\lg M / D$ & $\kappa$ & Progressive & 98.3 & 33 & Pos & 2/B & 68 & no & 1 \\
\hline 22 & 83 & $\mathrm{~F}$ & $\lg M / D$ & $\lambda$ & Progressive & 97.6 & 86 & Neg & $0 / A$ & 97 & no & 2 \\
\hline 23 & 72 & $M$ & $\lg M / D$ & $\kappa$ & Progressive & 96.6 & 16 & Neg & $4 / C$ & 81 & no & 2 \\
\hline 24 & 76 & M & $\lg M$ & $\lambda$ & Progressive & 94.3 & 0.6 & Neg & $0 / A$ & 158 & yes & 2 \\
\hline 25 & 68 & $M$ & $\lg M / D$ & $\lambda$ & Progressive & 93.5 & 0.4 & Pos & $0 / A$ & 163 & no & 2 \\
\hline 26 & 80 & $\mathrm{~F}$ & $\lg G$ & $\lambda$ & Progressive & 91.2 & 0.4 & Neg & $0 / A$ & 222 & no & 2 \\
\hline 27 & 78 & $\mathrm{~F}$ & $\lg M / D$ & $\lambda$ & Progressive & 88.5 & 100 & Neg & $0 / A$ & 180 & yes & 1 \\
\hline 28 & 73 & $M$ & $\lg M / D$ & $\lambda$ & Progressive & ND & 53 & Neg & $0 / A$ & 81 & no & 1 \\
\hline 29 & 62 & $\mathrm{~F}$ & $\lg M / D$ & $\lambda$ & Stable & 99.7 & 3.7 & Pos & O/A & 44 & no & 1 \\
\hline 30 & 78 & M & $\lg M / D$ & $\kappa / \lambda$ & Stable & 97.0 & 46.0 & Neg & $0 / A$ & 57 & no & 1 \\
\hline 31 & 69 & $\mathrm{~F}$ & $\lg M / D$ & $\kappa$ & Stable & 96.7 & 0.7 & Pos & $0 / A$ & 84 & no & 2 \\
\hline 32 & 68 & $\mathrm{~F}$ & $\lg M / D$ & $\kappa$ & Stable & 94.9 & 0.3 & Neg & $0 / A$ & 91 & no & 1 \\
\hline 33 & 62 & M & $\lg M / D$ & $\lambda$ & Stable & 94.7 & 0.4 & Neg & $0 / A$ & 73 & no & 2 \\
\hline 34 & 70 & M & $\lg M / D$ & $\kappa$ & Stable & 93.7 & 31.0 & Pos & $0 / A$ & 58 & no & 1 \\
\hline 35 & 62 & $M$ & $\lg M / D$ & $\lambda$ & Stable & 93.2 & 1.1 & $\mathrm{Neg}$ & O/A & 228 & no & 2 \\
\hline 36 & 74 & M & $\lg M / D$ & $\kappa$ & Stable & 92.5 & 1.0 & Pos & $2 / A$ & 125 & no & 2 \\
\hline 37 & 61 & M & $\lg M / D$ & $\lambda$ & Stable & 89.5 & 1.0 & Pos & $0 / A$ & 182 & no & 2 \\
\hline 38 & 61 & $\mathrm{~F}$ & $\lg G$ & $\kappa$ & Stable & 88.7 & 0.1 & Neg & $1 / \mathrm{A}$ & 182 & no & 1 \\
\hline 39 & 74 & M & $\lg M / D$ & $\kappa$ & Stable & 87.7 & 3.0 & Pos & $0 / A$ & 95 & no & 1 \\
\hline 40 & 73 & $\mathrm{~F}$ & $\lg M$ & $\kappa$ & Stable & ND & 0.8 & Pos & $0 / A$ & 35 & no & 1 \\
\hline
\end{tabular}

AShown as percentage similarity to the closest germline gene. ${ }^{B}$ According to criteria previously described by Rai et al. (40) and Binet et al. (41). ${ }^{C}$ Resulting from CLL-related events. DNumber of protein spots revealed on silver-stained 2-DE gels: 1, single left protein spot; 2, right and left protein spots. HC, heavy chain; LC, light chain; pos, positive as determined by immunoblot analysis; neg, negative as determined by immunoblot analysis; ND, not determined.

\section{Results}

2-DE identifies distinct patterns of protein expression in CLL patient subsets. To minimize interpatient variations, we started by investigating a limited number of CLL patients who were completely concordant for $\operatorname{Ig} V_{H}$ mutational status (unmutated vs. mutated), CD38 expression (positive vs. negative), and clinical behavior (progressive vs. stable disease) (Table 1, patients 1-14). Seven patients carried unmutated $\operatorname{Ig} V_{H}$ genes, were positive for CD38, and experienced clinical progression requiring treatment during their clinical course (subset with poor prognoses). The other 7 patients had mutated $\operatorname{Ig} V_{H}$ genes, were negative for CD38, and had stable disease throughout a long follow-up period (median time, 102 months) (subset with good prognoses).
$\mathrm{CD}_{19}{ }^{+} \mathrm{CD}^{+}$purified leukemic cells were lysed and proteins resolved on 2-DE and visualized by silver staining. The protein profile analysis on silver-stained gels showed a number of protein spots differentially expressed in the samples obtained from the 2 CLL subsets. We focused our attention on 2 close protein spots with the same relative molecular mass $(\mathrm{Mr})$ of $79 \mathrm{kDa}$ and different isoelectric points (pIs; 4.83 and 4.86; Figure 1A) that were both expressed in the 2-DE gels obtained from most patients with good prognoses (Figure 1B and Table 1) while the more acidic protein prevailed in the preparations from the patients with poor prognoses (Figure $1 \mathrm{~B}$ and Table 1). Though the number of patients studied was small, 5 of 7 progressive but only 1 of 7 stable patients had 1 protein spot. Differences in protein expression pattern were statistically significant $\left(P<0.05\right.$ using a $\chi^{2}$ test). 
MS analysis identifies HS1 as the protein differentially expressed in the 2 subsets of CLL patients. The 2 protein spots were excised from 2-DE gels and analyzed by matrix-assisted laser desorption/ionization-time-of-flight (MALDI-TOF) MS, after tryptic digestion. The 2 protein spots resulted from the same protein and were identified as HS1 (see Supplemental Table 1; available online with this article; doi:10.1172/JCI24276DS1). Their resulting from the same protein suggested that the protein spots represent either structural or functional isoforms of the same protein. To confirm the identification results, 2-DE gels from 5 representative patients were transferred to nitrocellulose and incubated with a monoclonal anti-HS1 antibody that hybridized specifically at the same mass values and pIs of both protein spots (Figure 1C).

Western blot analysis on SDS-PAGE gels was performed with anti-HS1 to define the expression levels of the molecule irrespective of its functional modifications. This analysis showed that the protein was expressed in equal amounts in both CLL subsets as were the signaling molecules Syk and Lyn $(11,12)$ (data not shown).

Treatment of CLL cell lysates with phosphatase demonstrates the presence of phosphorylation on the differentially expressed HS1 protein spots. The different pIs of the 2 (left and right) HS1 protein spots (Figure 1) suggested the possibility that posttranslational modifications might be responsible for the differential expression of HS1 revealed by 2-DE.

As it has been shown that HS1 may become phosphorylated at several tyrosine residues following BCR stimulation (24-27), resulting in a more acidic $\mathrm{pI}$, we aimed at determining whether the different HS1 migration pattern in 2-DE might be caused by protein phospohorylation.

We performed dephosphorylation experiments using $\lambda$ protein phosphatase ( $\lambda$ PPase), which removes phosphoryl groups from different residues (Tyr, Ser, Thr, and His) (28). CLL cell lysates from purified cells of 4 representative patients ( 2 from each group) were incubated in the presence of $\lambda$ PPase using different incubation times. After 7 and 15 hours of incubation, 2-DE and Western blot with anti-HS1 antibody (Figure 2) revealed a shift of the more acidic isoforms toward a less acidic pI. This documents that the HS1 left protein spot is a phosphorylated form of the protein and that the HS1 right protein spot corresponds to a dephosphorylated molecule (Figure 2). Hence, these data indicate that most of the HS1 protein present in the cells from CLL patients with poor prognoses is constitutively phosphorylated whereas only a fraction of HS1 is phosphorylated in the cells from patients with good prognoses.

In particularly well-resolved 2-DE, it was possible to detect several intermediate HS1 isoforms that probably corresponded to different rates of phosphorylation (Figure 2). These protein spots following $\lambda$ PPase incubation shifted through successive intermediates from more acidic and heavy isoforms to less acidic and lighter isoforms (Figure 2).

HS1 expression pattern correlates with CD38 expression and $\operatorname{Ig} V_{H}$ mutational status in a larger cohort of CLL patients. We expanded on the data obtained from the above-described group of patients by examining the expression of HS1 in 26 further unselected patients who were more heterogeneous in terms of biological prognostic factors and clinical courses (Table 1, patients 15-40). Overall, in the 40 patients studied for HS1 expression (Table 1), the expression of CD38 had a significant correlation with $\operatorname{Ig} V_{H}$ mutational status $(P<0.001)$, and the expression of ZAP-70 correlated with $\operatorname{Ig} V_{H}$ mutational status $(P=0.003)$. In these patients, the differential pattern of HS1 expression (Table 1) was found to correlate with both the expression of CD38 $(P=0.022)$ and $\operatorname{Ig} V_{H}$ mutational status $(P=0.008)$. Rather unexpectedly, no significant correlation was found with the expression of ZAP-70 $(P=0.505)$. When the 40 patients were grouped according to HS1 profile, it appeared that those presenting 1 single HS1 protein spot had a median survival time (184 months) significantly shorter than those with 2 protein spots (median survival not reached; $P=0.024$; Figure 3 ).

Modifications of Syk and HS1 upon stimulation of the BCR in leukemic and normal $B$ cells. CLL cells respond differently to BCR stimulation, depending on their biological features, with cells of unmutated and/or CD38- and/or ZAP-70-positive patients being better responders than cells of mutated and/or CD38- and/or ZAP-70negative patients $(11,12,16,22,23)$. Therefore, we planned to investigate whether an antigen-like BCR stimulation might modify the phosphorylation status of Syk and HS1 molecules involved in the downstream signaling pathway.

First, we studied the phosphorylation status of Syk ex vivo in 10 of 14 fully concordant CLL patients (Table 1, patients 1-14), after an in vitro culture of 5 minutes and immunoprecipitation. The levels of phosphorylation of Syk protein were constitutively similar in all patients irrespective of their biological features. After in vitro stimulation for 5 minutes with anti-IgM antibodies, 4 of 5 unmutated, CD38-positive cases increased the phosphorylated form of the protein, while no mutated, CD38-negative cases did so (data not shown), confirming previously published results $(11,12,22)$.

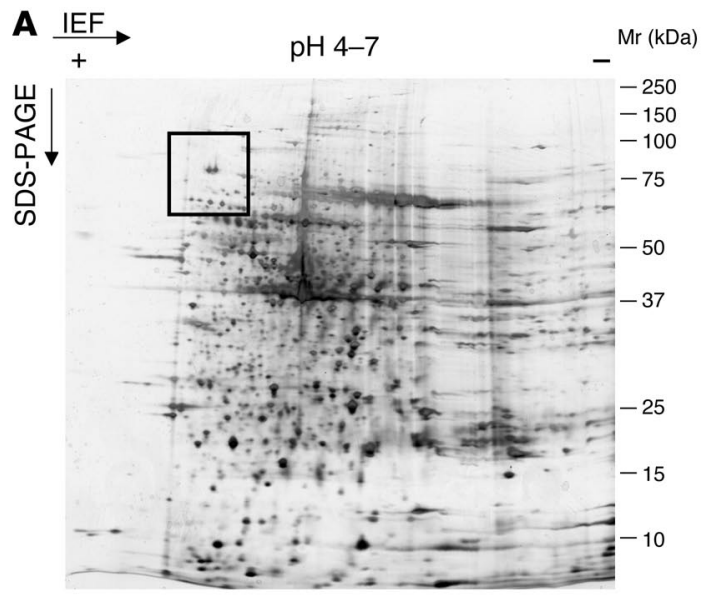

\section{Figure 1}

2-DE proteomic analysis of purified CLL cells. (A) The square identifies 2 close protein spots with the same Mr of $79 \mathrm{kDa}$ and different pls, 4.83 and 4.86, which were identified as HS1 proteins by MALDI-TOF MS analysis (see Results). IEF, isoelectric focusing. (B) Both right and left protein spots were expressed in 2D gels obtained from 2 representative CLL patients with good prognoses (subset 1 ) while only the more acidic one was present in 2D gels obtained from 2 representative CLL patients with poor prognoses (subset 2). Arrows indicate the left and right protein spots identified as HS1. (C) Proteins obtained from 5 representative CLL patients were resolved on 2-DE that was either silver stained (left panel) or transferred onto nitrocellulose and incubated with an anti-HS1 antibody (right panel). One representative case is shown. The antibody hybridizes in an area corresponding to the protein spots of interest. WB, Western blot. 
Subset 1
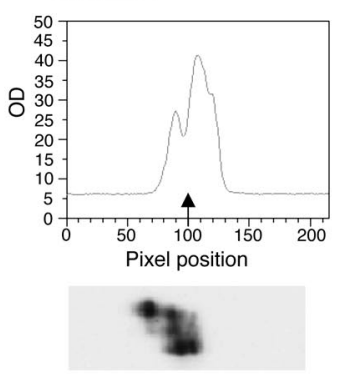

$-\lambda$ PPase

Subset 2
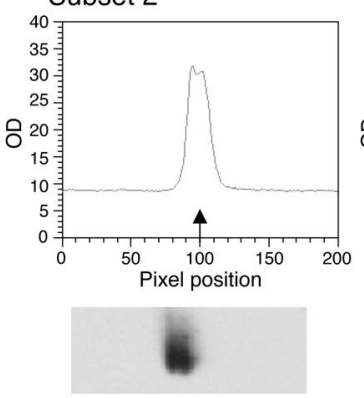
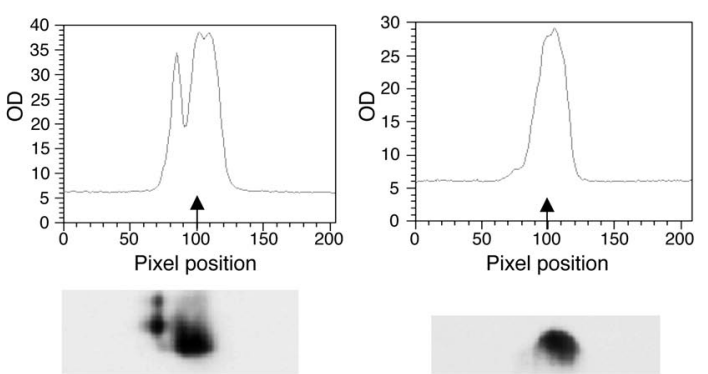

$+(7 \mathrm{~h}) \lambda \mathrm{PPase}$
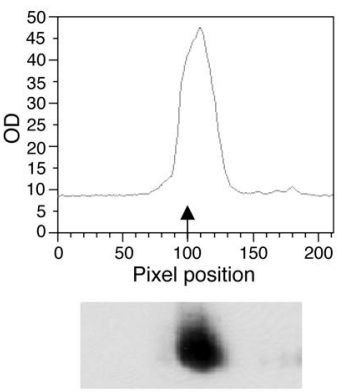

\section{Figure 2}

The more acidic protein spot is a phosphorylated form of HS1 protein. Cells from 4 CLL patients belonging to subsets 1 and 2 were treated with $\lambda$ PPase; proteins were resolved on 2-DE, transferred onto nitrocellulose, and incubated with a specific anti-HS1 antibody at different time points. One representative case is shown for each subset. The densitometric analysis indicates the progressive shift of the HS1 protein from a more to a less acidic form after 7 and 15 hours of incubation.
To investigate whether HS1 protein could be modified after similar antigen-like BCR stimulation, both CLL leukemic and normal mature B lymphocytes purified from tonsils were cultured for 2 minutes in the presence or absence of anti-IgM and then immediately harvested for protein separation. To allow the evaluation of potential modifications of the phosphorylated forms, we selected 4 CLL patients with 2 protein spots, 2 mutated, CD38-negative patients, and 2 unmutated, CD38-positive patients. In these experimental conditions, the capacity of CLL cells to increase phosphorylation levels of HS1 were variable. As expected $(11,12)$, the 2 mutated, CD38-negative patients did not modify their 2-protein spots pattern, while 1 of 2 unmutated, CD38-positive patients with 2 protein spots showed an increase in the phosphorylated left protein spot (data not shown). In normal B cells, 2-DE showed a shift of the protein spot representing the non- or less-phosphorylated form of HS1 (right protein spot) toward a more acidic position (left protein spot) that corresponded to the highly phosphorylated form (Figure 4A).

We then analyzed the proteomic profile of ex vivo, purified, naive, and memory tonsillar B lymphocytes (purity greater than 98\%), which differ in their in vivo experience with regard to encountering antigens. Naive B cells showed the presence of both HS1 forms (left and right protein spots) at equal levels while memory B cells expressed mainly the phosphorylated fraction (left protein spot; Figure 4B). This confirms that in vivo antigen activation may be responsible for the differential aspect of HS1 migration patterns in 2-DE

\section{Figure 3}

CLL patients with $1 \mathrm{HS} 1$ protein spot have a shorter cumulative survival time. Kaplan-Meier curves show cumulative survival of CLL patients grouped according to HS1-expression pattern (1 single left protein spot vs. 2 protein spots). Patients with 2 protein spots (filled circles) experienced a significantly longer survival period (median survival not reached) than those with only 1 (open circles; median survival, 184 months; $P=0.024$ ). gels. Western blot analysis of protein lysates obtained from both naive and memory B cells showed similar levels of total HS1 protein (data not shown), regardless of their functional modification.

\section{Discussion}

The use of gene expression profiling has shown that CLL cases, irrespective of Ig mutational status, are characterized by a common gene expression signature $(23,29)$, which suggests that all cases of CLL share a common mechanism of transformation and/or cell of origin. That notwithstanding, CLL is heterogeneous at both clinical and biological levels. CLL patients can be grouped into different subsets on the basis of biological features that have been shown to predict the clinical behavior (aggressive vs. indolent) of their disease. A number of these features are related to differences in cell capacity to respond to signals originating in the microenvironment. A prominent example is the presence of somatic hypermutations of $\operatorname{Ig} V_{H}$ genes, which reveals the stimulation of $\operatorname{BCR}(2,10)$.

In this work, we first analyzed a limited number of CLL patients, purposely selected at the 2 extremes of a Gaussian curve to mini-

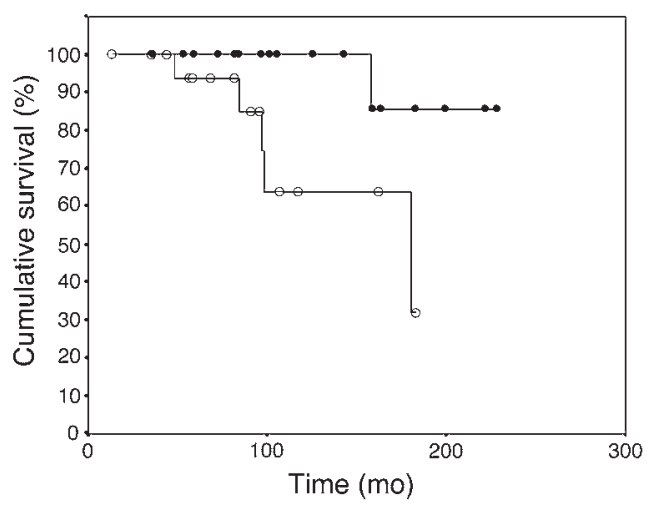


A
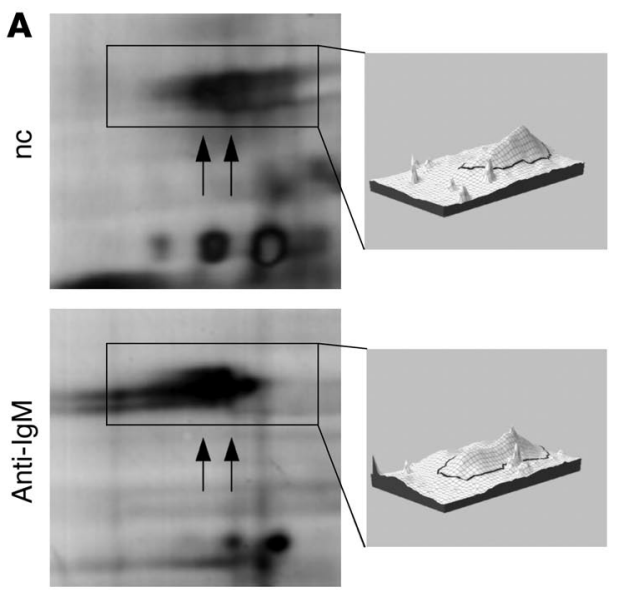
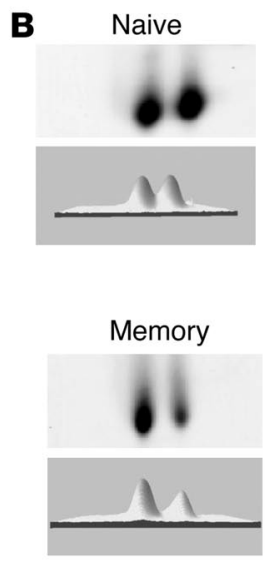

\section{Figure 4}

HS1 pattern of expression in normal B lymphocytes. (A) Purified tonsillar B cells were cultured in the presence or absence of anti$\mathrm{IgM}$ antibody for 2 minutes. Proteins from untreated and treated cells were resolved on 2-DE and revealed by silver staining. The arrows indicate the $2 \mathrm{HS} 1$ protein spots, and 3D-densitometric analysis (right panels) shows a shift from a less acidic to a more acidic form of HS1 protein following BCR stimulation. nc, negative control. (B) Proteins obtained from FACS-purified naive and memory tonsillar B cells were resolved on 2-DE and revealed by silver staining. The top panel of each B cell population identifies the different HS1 forms. The 3D-densitometric analysis shows that both protein spots are expressed in similar amounts in naive B cells, while in memory B cells, the more acidic form is predominantly represented. mize interpatient variations. The patients were grouped into 2 subsets (those with good prognoses and those with poor prognoses) clustered on the basis of the complete concordance of biological factors $\left(\operatorname{Ig} V_{H}\right.$ mutational status and CD38 expression) and clinical outcome (stable or progressive disease). This approach allowed us to show that the features of the protein HS1 significantly differed between the 2 groups, as most HS1 protein detected in the cells from patients with poor prognoses was constitutively phosphorylated whereas only a minor fraction in the cells of patients with good prognoses was phosphorylated (Figure 1). This finding was confirmed by adding to the study a larger unselected group of 26 patients. Overall the proteomic pattern of HS1 maintained a significant correlation with both the $\operatorname{Ig} V_{H}$ mutational status and the expression of CD38. The rather unexpected lack of correlation with the expression of ZAP-70 may simply reflect the size of the cohort of patients studied. However, given the functional role of ZAP-70, this requires further investigation. Of interest, when the 40 CLL patients studied were pooled together and their survival curves were related to their HS1 profiles, the patients presenting a single left HS1 protein spot on the 2-DE gels - and thus showing a predominately phosphorylated form of HS1 - were shown to have a median survival significantly shorter than that of those presenting 2 protein spots, i.e., having a predominately unphosphorylated form of HS1 (Figure 3).

HS1 is a $79-\mathrm{kDa}$ intracellular protein expressed exclusively in cells of lymphohemopoietic origin. In B and T lymphocytes, it plays a relevant role in signal transduction through surface antigen receptors $(24,30)$. In B lymphocytes, it undergoes rapid phosphorylation at several tyrosine residues following BCR stimulation (24-26) and is an important substrate of protein tyrosine kinases such as Syk and Src family proteins, including Lyn, Fgr, Fyn, and Lck. Studies of targeted mice have revealed that HS1 has a central role in B cell responsiveness, as HS1-deficient mice exhibit defective antigen-induced clonal expansion and deletion (31).

We found that in vitro and in vivo stimulation of normal B cells through the BCR is sufficient for phosphorylating HS1 protein. This finding is well in keeping with the observation that, in normal T lymphocytes (32), costimulation activates the same signaling molecules triggered by antigen-receptor stimulation but does not lead to phosphorylation of HS1. These observations indicate that the phosphorylation of HS1 is induced by the stimulation of antigen receptor and suggest that in B cells HS1 phosphorylation can be considered a signature of BCR engagement. Therefore, our findings that in malignant CLL cells from the group of patients with poor prognoses, most HS1 protein is in the phosphorylated form suggest the presence of an ongoing stimulation likely triggered by a recent/persistent BCR-mediated activation. This possibility is supported by the fact that in vitro anti-IgM stimulation may induce phosphorylation of Syk in patients with poor prognoses (see also refs. 11, 12). These data reinforce the concept that emerged from the study of $\operatorname{Ig}$ gene rearrangement features $(5,33$, $34)$ - that the stimulation of BCR appears to have a relevant role in disease that behaves more aggressively. Taken together, these observations led us to postulate that circulating U-CLL cells maintain the full capacity to respond to a still-unknown triggering antigen to which they may be chronically exposed. The implication is that in CLL patients with poor prognoses, stimulation of BCR appears to be relevant not only for the onset but also for the maintenance and progression of the disease.

In contrast, patients with good prognoses tend to have HS1 in the nonphosphorylated form and are unable to phopsphorylate Syk upon anti-IgM stimulation. These features are in keeping with the notion that mutated cases show, at least in vitro, a characteristic BCR unresponsiveness $(11,12)$. This is generally considered a sign of an anergic state, probably induced after an antigen-like stimulation (35). This suggests that in patients with good prognoses, antigen stimulation occurred at the onset of the disease, but ongoing BCR stimulation is not necessary for its maintenance. Instead, other factors originating from the microenvironment (36), such as cytokines, might help maintain the stimulated clone.

In both good-prognosis and bad-prognosis patients, it remains to be determined which stimulus sustains BCR activation in patients, whether it is a foreign antigen or an autoantigen. Considering the skewing of Ig gene usage $(1,3,4)$ and the fact that a high proportion of CLL B cells display natural autoantibody activity (37), it follows that the most likely candidate is an autoantigen.

Taking all of these data into account, it appears that antigen stimulation may have a central role in the natural history of CLL and especially of U-CLL with a poor prognosis. The very fact that CLL with poor prognoses are characterized by the prominent expression of the phosphorylated form of HS1 indicates a potential area of study for determining new tools that may be applied at diagnosis in order to identify patients with disease that is likely to run a progressive course. More importantly, it suggests a new molecular target for the treatment of patients who present with aggressive disease. 


\section{Methods}

Tissue samples and cell purification. Leukemic lymphocytes were obtained from peripheral blood of 40 CLL patients, diagnosed according to National Cancer Institute Working Group (NCIWG) guidelines (38). The following parameters were analyzed for each patient: CD38 expression (39), $\operatorname{Ig} V_{H}$ mutational status (39), ZAP-70 expression (22), disease stage according to Binet (40) or modified Rai criteria (41), history of treatment, characterization of disease as progressive or stable as defined by the NCIWG (38), and survival time. All patients were either untreated or off therapy for at least 6 months before the beginning of the study.

Leukemic CD19+ cells were purified using a B lymphocyte-enrichment kit (RosetteSep; StemCell Technologies) following manufacturer's instructions. Purity of all preparations was always above $99 \%$, and the cells coexpressed CD19 and CD5 on their cell surfaces as checked by flow cytometry (FC500; Beckman Coulter); preparations were virtually devoid of NK and T lymphocytes. The purified cells, at a concentration of $25 \times 10^{6}$ (1 mg protein/sample, as checked by bicinchoninic acid assay), were washed with PBS, pelleted, and stored at $-20^{\circ} \mathrm{C}$.

Tonsillar B cells from discarded tissue were stained with anti-IgD FITC (SouthernBiotech), anti-CD19 PE, and anti-CD38 PC5 (Beckman Coulter), then FACS-purified on a Coulter Altra (Beckman Coulter) in order to obtain naive $\mathrm{B}$ cells $\left(\mathrm{CD} 19^{+}, \mathrm{IgD}^{+}\right.$, and $\left.\mathrm{CD} 38^{-}\right)$and memory B cells (CD19', $\mathrm{IGD}^{-}$, and $\mathrm{CD}^{-} 8^{-}$) (42). Purity was greater than $98 \%$.

All tissue samples were obtained with approval by the institutional review board of San Raffaele University Hospital (Milan, Italy).

Cell culture and stimulation. Purified normal and leukemic B cells were cultured in RPMI 1640 medium supplemented with $10 \%$ FCS, 2 mM L-glutamine and $15 \mu \mathrm{g} / \mathrm{ml}$ gentamicin (complete RPMI; Invitrogen Corp., Life Technologies Inc.) at a concentration of $3 \times 10^{6} \mathrm{cells} / \mathrm{ml}$ in the presence or absence of goat $\mathrm{F}\left(\mathrm{ab}^{1}\right) 2$ anti-human IgM antibodies $(20 \mu \mathrm{g} / \mathrm{ml}$; CALTAG Laboratories) for 2 or 5 minutes. Cells were then lysed, pelletted, and stored at $-80^{\circ} \mathrm{C}$.

Monodimensional electrophoresis and 2-DE. The pellets were lyophilized and solubilized with 2-DE buffer (9 M Urea; $10 \mathrm{mM}$ Tris; 4\% 3-[(3-cholamid opropyl)dimethylammonio]-1-propanesulfonate [CHAPS]; 65 mM DTT; 2\% IPG buffer ampholine, $\mathrm{pH}$ 4-7 [Amersham Biosciences]; and protease inhibitor cocktail). Protein samples, $1 \mathrm{mg}$ or $250 \mathrm{mg}$, were applied to 18or 7-cm IPGstrips, pH 4-7 (Amersham Biosciences), respectively, by in-gel rehydration. Isoelectric focusing was performed with an IPGphor system (Amersham Biosciences) following standard protocol as described by Conti et al. (43). Strips were equilibrated in $50 \mathrm{mM} \mathrm{pH} 8.8$ Tris-HCl buffer containing $6 \mathrm{M}$ urea, $30 \%$ glycerol, $2 \% \mathrm{SDS}$, and $2 \% \mathrm{DTT}$, followed by an incubation in the same buffer replacing DTT with $2.5 \%$ iodoacetamide. The strips were loaded on the top of either 9-16\% gradient or $10 \%$ acrylamide SDS-PAGE gels for the second dimension separation.

Protein spots were made visible by staining gels with MS compatible silver stain (44). Images were acquired at high resolution using a Molecular Dynamics Personal SI Laser Densitometer (Amersham Biosciences) and 2-DE protein patterns analyzed using ImageMaster 2D Platinum 5.0 software (Amersham Biosciences) or Progenesis Workstation, version 2004 (Nonlinear Dynamics). Mrs were estimated by comparison with Mr reference markers (Bio-Rad Laboratories) and $\mathrm{PI}$ values assigned to detected protein spots by calibration as described in the Amersham Biosciences guidelines.

Monodimensional SDS-PAGE was performed after solubilizing the cell pellets with Laemmli buffer and loading $10 \mu \mathrm{g}$ of protein into $10 \%$ acrylamide gel.

Immunoprecipitation and Western blot analysis. Total cell lysates were incubated overnight at $4{ }^{\circ} \mathrm{C}$ with antiphosphotyrosine antibody $4 \mathrm{G} 10$ (Upstate Biotechnology) immobilized on protein G-coated sepharose beads (Amersham Biosciences). After a quick spin, the supernatant was collected, and the beads were washed 3 times with lysis buffer. Immunocomplexes were recovered by incubation with Laemmli buffer $\left(5\right.$ minutes at $\left.95^{\circ} \mathrm{C}\right)$.

Immunoprecipitated or whole proteins from cells incubated with or without anti-IgM stimulation were resolved either by SDS-PAGE or 2-DE, electron-transferred onto nitrocellulose membranes, and incubated 2 hours with 1 of the following antibodies (1:500 to 1:1000 working dilution): monoclonal anti-HS1 (Transduction Laboratories; BD Biosciences - Pharmingen), monoclonal anti-Syk, rabbit polyclonal anti-Lyn (Upstate Biotechnology), and anti- $\beta$-actin (Sigma-Aldrich). Immunoreactivity was revealed by incubation with either goat anti-rabbit Ig or rabbit anti-mouse Ig conjugated with HRP (Upstate Biotechnology), followed by ECL reaction (Amersham Biosciences) and film exposures.

Protein identification by MALDI-TOF MS analysis. Protein spots of interest were excised from gels, either by manual or automated excision (ProXCISION; PerkinElmer), reduced, alkylated, and digested overnight with bovine trypsin (Roche Diagnostics Corp.) as previously described (45). Aliquots of the supernatant $(1 \mu \mathrm{l})$ were used for MS analysis by the dried-droplet technique, using $\alpha$-cyano-4-hydroxycinnamic acid as a matrix. Mass spectra were obtained using a MALDI-TOF Voyager-DE STR (Applied Biosystems) mass spectrometer. Alternatively, gel fragments were further extracted and the resulting peptide mixture subjected to a single desalting/concentration step before MS analysis over $\mu$ ZipTipC18 (Millipore). Spectra were internally calibrated using trypsin autolysis products and processed via Data Explorer software (version 3.2; Applied Biosystems). Proteins were unambiguously identified by searching the comprehensive nonredundant protein databases of the National Center for Biotechnology Information (NCBI, http://www.ncbi.nlm.nih.gov/entrez/query.fcgi?db=Protein) and the Mass Spectrometry protein sequence DataBase (MSDB, http://csc-fserve.hh.med. ic.ac.uk/msdb.html) selected by default by the software programs ProFound (http://prowl.rockefeller.edu/profound_bin/WebProFound.exe; ref. 46) and Mascot (http://www.matrixscience.com; ref. 47), respectively. One missed cleavage per peptide was allowed, and an initial mass tolerance of 50 parts per million was used in all searches.

Phosphatase treatment. $\lambda$ PPase (New England Biolabs Inc.) treatment was performed as described by Yamagata et al. (28) with modifications. In brief, pelleted cells $\left(25 \times 10^{6}\right)$ were resuspended for 12 hours in lysis buffer $(1 \%$ w/v NP-40, 1\% w/v SDS, $50 \mathrm{mM}$ Tris $\mathrm{pH} 7.6$, and $150 \mathrm{mM} \mathrm{NaCl}$ protease inhibitor cocktail). We brought $60 \mu \mathrm{l}$ of lysate, corresponding to $600 \mu \mathrm{g}$ of protein, to a final volume of $600 \mu \mathrm{l}$ with deionized water, then added $20 \mu \mathrm{l}$ of $20 \mathrm{mM} \mathrm{MnCl}_{2}$ solution and $20 \mu \mathrm{l}$ of $\lambda$ PPase buffer. After each addition, the solution was gently mixed. The mixture was divided into 2 aliquots, and 300 units of $\lambda$ PPase was added to 1 of the aliquots. After mixing, aliquots were incubated for 7 hours or 15 hours at $30^{\circ} \mathrm{C}$. Proteins were acetone precipitated at $-20^{\circ} \mathrm{C}$ and used for 2-DE analysis.

Statistical analysis. Fisher exact test (2-tailed) was performed to determine the degree of association among patients' subsets (with mutated or unmutated $\operatorname{Ig} V_{H}$ genes, negative or positive for CD38, negative or positive for ZAP-70, with 1 or 2 HS1 protein spots). Cumulative survival analysis was obtained according to the Kaplan-Meier method.

\section{Acknowledgments}

This work was supported in part by the Associazione Italiana per la Ricerca sul Cancro (AIRC), the Progetto Oncologia Centro Nazionale delle Ricerche-Ministero dell' Istruzione dell' Università e della Ricerca (CNR-MIUR), the Ministero dell' Università e della Ricerca Scientifica e Tecnologica (MURST), the Ministero della Sanità (Ricerca Finalizzata 2002, no.183), the Fondazione Cariplo, and the MIUR Fondi per gli Investimenti della Ricerca di Base (MIUR-Firb; RBNE01LNX7-006). We are grateful to Mary Carletti for her valuable technical assistance and to Michela Frenquelli and Annalisa Camporeale for their continuous support in lab work. We 
are indebted to Michele De Palma and Nadia Coltella for their help in performing experiments and for helpful discussion. We also want to thank all colleagues working in the Laboratory of Cancer Immunology at the Institute for Cancer Research and Treatment and the Proteome Biochemistry Unit at the San Raffaele Scientific Institute for their friendly and collaborative support.

Received for publication December 21, 2004, and accepted in revised form April 26, 2005.

1. Fais, F., et al. 1998. Chronic lymphocytic leukemia B cells express restricted sets of mutated and unmutated antigen receptors. J. Clin. Invest. 102:1515-1525.

2. Hamblin, T.J., Davis, Z., Gardiner, A., Oscier, D.G., and Stevenson, F.K. 1999. Unmutated $\operatorname{Ig} V(\mathrm{H})$ genes are associated with a more aggressive form of chronic lymphocytic leukemia. Blood. 94:1848-1854.

3. Kipps, T.J., et al. 1989. Developmentally restricted immunoglobulin heavy chain variable region gene expressed at high frequency in chronic lymphocytic leukemia. Proc. Natl. Acad. Sci. U. S. A. 86:5913-5917.

4. Schroeder, H.W., Jr., and Dighiero, G. 1994. The pathogenesis of chronic lymphocytic leukemia: analysis of the antibody repertoire. Immunol. Today. 15:288-294.

5. Ghia, P., et al. 2005. Geographical patterns and pathogenetic implications of IGHV gene usage in chronic lymphocytic leukemia: the lesson of the IGHV3-21 gene. Blood. 105:1678-1685.

6. Tobin, G., et al. 2004. Subsets with restricted immunoglobulin gene rearrangement features indicate a role for antigen selection in the development of chronic lymphocytic leukemia. Blood. 104:2879-2885.

7. Widhopf, G.F., 2nd, et al. 2004. Chronic lymphocytic leukemia B cells of more than $1 \%$ of patients express virtually identical immunoglobulins. Blood. 104:2499-2504.

8. Messmer, B.T., et al. 2004. Multiple distinct sets of stereotyped antigen receptors indicate a role for antigen in promoting chronic lymphocytic leukemia. J. Exp. Med. 200:519-525.

9. Damle, R.N., et al. 2002. B-cell chronic lymphocytic leukemia cells express a surface membrane phenotype of activated, antigen-experienced B lymphocytes. Blood. 99:4087-4093.

10. Damle, R.N., et al. 1999. Ig V gene mutation status and CD38 expression as novel prognostic indicators in chronic lymphocytic leukemia. Blood. 94:1840-1847.

11. Chen, L., et al. 2002. Expression of ZAP-70 is associated with increased B-cell receptor signaling in chronic lymphocytic leukemia. Blood. 100:4609-4614.

12. Lanham, S., et al. 2003. Differential signaling via surface IgM is associated with VH gene mutational status and CD38 expression in chronic lymphocytic leukemia. Blood. 101:1087-1093.

13. Vilen, B.J., Nakamura, T., and Cambier, J.C. 1999. Antigen-stimulated dissociation of BCR mIg from Ig-alpha/Ig-beta: implications for receptor desensitization. Immunity. 10:239-248.

14. Deaglio, S., et al. 2003. CD38 is a signaling molecule in B-cell chronic lymphocytic leukemia cells. Blood. 102:2146-2155.

15. Hamblin, T.J., et al. 2002. CD38 expression and immunoglobulin variable region mutations are independent prognostic variables in chronic lymphocytic leukemia, but CD38 expression may vary during the course of the disease. Blood. 99:1023-1029.

16. Zupo, S., et al. 1996. CD38 expression distinguishes two groups of B-cell chronic lymphocytic leukemias with different responses to anti-IgM
Address correspondence to: Federico Caligaris-Cappio, Department of Oncology, Università Vita e Salute-San Raffaele, Via Olgettina 58, 20132 Milan, Italy. Phone: 39-02-26432390; Fax: 39-02-26410154; E-mail: caligaris.federico@hsr.it. Or to: Massimo Alessio, Proteome Biochemistry Unit, San Raffaele Scientific Institute, Via Olgettina 58, 20132 Milan, Italy. Phone: 39-02-26434725; Fax: 39-02-26434153; E-mail: alessio.massimo@hsr.it.

\section{Cristina Scielzo and Paolo Ghia contributed equally to this work}

antibodies and propensity to apoptosis. Blood. 88: $1365-1374$.

17. Zupo, S., et al. 2000. Apoptosis or plasma cell differentiation of CD38-positive B-chronic lymphocytic leukemia cells induced by cross-linking of surface IgM or IgD. Blood. 95:1199-1206.

18. Chen, L., et al. 2005. ZAP-70 directly enhances IgM signaling in chronic lymphocytic leukemia. Blood. 105:2036-2041.

19. Chan, A.C., Iwashima, M., Turck, C.W., and Weiss, A. 1992. ZAP-70: a $70 \mathrm{kd}$ protein-tyrosine kinase that associates with the TCR zeta chain. Cell. 71:649-662.

20. Crespo, M., et al. 2003. ZAP-70 expression as a surrogate for immunoglobulin-variable-region mutations in chronic lymphocytic leukemia. N. Engl. J. Med. 348:1764-1775.

21. Rassenti, L.Z., et al. 2004. ZAP-70 compared with immunoglobulin heavy-chain gene mutation status as a predictor of disease progression in chronic lymphocytic leukemia. N. Engl. J. Med. 351:893-901.

22. Wiestner, A., et al. 2003. ZAP-70 expression identifies a chronic lymphocytic leukemia subtype with unmutated immunoglobulin genes, inferior clinical outcome, and distinct gene expression profile. Blood. 101:4944-4951.

23. Rosenwald, A., et al. 2001. Relation of gene expression phenotype to immunoglobulin mutation genotype in B cell chronic lymphocytic leukemia. J. Exp. Med. 194:1639-1647.

24. Yamanashi, Y., et al. 1993. Identification of HS1 protein as a major substrate of protein-tyrosine kinase(s) upon B-cell antigen receptor-mediated signaling. Proc. Natl. Acad. Sci. U. S. A. 90:3631-3635.

25. Yamanashi, Y., et al. 1997. Role of tyrosine phosphorylation of HS1 in B cell antigen receptormediated apoptosis. J. Exp. Med. 185:1387-1392.

26. Brunati, A.M., et al. 1999. Molecular features underlying the sequential phosphorylation of HS1 protein and its association with c-Fgr protein-tyrosine kinase. J. Biol. Chem. 274:7557-7564.

27. Ruzzene, M., Brunati, A.M., Marin, O., DonellaDeana, A., and Pinna, L.A. 1996. SH2 domains mediate the sequential phosphorylation of HS1 protein by $\mathrm{p} 72$ syk and Src-related protein tyrosine kinases. Biochemistry. 35:5327-5332.

28. Yamagata, A., et al. 2002. Mapping of phosphorylated proteins on two-dimensional polyacrylamide gels using protein phosphatase. Proteomics. 2:1267-1276.

29. Klein, U., et al. 2001. Gene expression profiling of B cell chronic lymphocytic leukemia reveals a homogeneous phenotype related to memory B cells. J. Exp. Med. 194:1625-1638.

30. Hata, D., et al. 1994. Tyrosine phosphorylation of MB-1, B29, and HS1 proteins in human B cells following receptor crosslinking. Immunol. Lett. 40:65-71.

31. Taniuchi, I., et al. 1995. Antigen-receptor induced clonal expansion and deletion of lymphocytes are impaired in mice lacking HS1 protein, a substrate of the antigen-receptor-coupled tyrosine kinases. EMBO J. 14:3664-3678.

32. Hutchcroft, J.E., Slavik, J.M., Lin, H., Watanabe,
T., and Bierer, B.E. 1998. Uncoupling activationdependent HS1 phosphorylation from nuclear factor of activated $\mathrm{T}$ cells transcriptional activation in Jurkat T cells: differential signaling through CD3 and the costimulatory receptors $\mathrm{CD} 2$ and CD28. J. Immunol. 161:4506-4512.

33. Tobin, G., et al. 2002. Somatically mutated Ig $\mathrm{V}(\mathrm{H}) 3-21$ genes characterize a new subset of chronic lymphocytic leukemia. Blood. 99:2262-2264.

34. Tobin, G., et al. 2003. Chronic lymphocytic leukemias utilizing the VH3-21 gene display highly restricted Vlambda2-14 gene use and homologous CDR3s: implicating recognition of a common antigen epitope. Blood. 101:4952-4957.

35. Goodnow, C.C. 1992. B-cell tolerance. Curr. Opin. Immunol. 4:703-710.

36. Ghia, P., and Caligaris-Cappio, F. 2000. The indispensable role of microenvironment in the natural history of low-grade B-cell neoplasms [review]. Adv. Cancer Res. 79:157-173.

37. Caligaris-Cappio, F. 1996. B-chronic lymphocytic leukemia: a malignancy of anti-self B cells. Blood. 87:2615-2620

38. Cheson, B.D., et al. 1996. National Cancer Institute-sponsored Working Group guidelines for chronic lymphocytic leukemia: revised guidelines for diagnosis and treatment. Blood. 87:4990-4997.

39. Ghia, P., et al. 2003. The pattern of CD38 expression defines a distinct subset of chronic lymphocytic leukemia (CLL) patients at risk of disease progression. Blood. 101:1262-1269.

40. Binet, J.L., et al. 1977. A clinical staging system for chronic lymphocytic leukemia: prognostic significance. Cancer. 40:855-864.

41. Rai, K.R., and Han, T. 1990. Prognostic factors and clinical staging in chronic lymphocytic leukemia. Hematol. Oncol. Clin. North Am. 4:447-456.

42. Ghia, P., et al. 1998. Unbalanced expression of bcl-2 family proteins in follicular lymphoma: contribution of CD40 signaling in promoting survival. Blood. 91:244-251.

43. Conti, A., et al. 2004. Proteome study of human cerebrospinal fluid following traumatic brain injury indicates fibrin(ogen) degradation products as trauma-associated markers. J. Neurotrauma. 21:854-863.

44. Mortz, E., Krogh, T.N., Vorum, H., and Gorg, A. 2001. Improved silver staining protocols for high sensitivity protein identification using matrixassisted laser desorption/ionization-time of flight analysis. Proteomics. 1:1359-1363.

45. Shevchenko, A., Wilm, M., Vorm, O., and Mann, M. 1996. Mass spectrometric sequencing of proteins silver-stained polyacrylamide gels. Anal. Chem. 68:850-858.

46. Zhang, W., and Chait, B.T. 2000. ProFound: an expert system for protein identification using mass spectrometric peptide mapping information. Anal. Chem. 72:2482-2489.

47. Perkins, D.N., Pappin, D.J., Creasy, D.M., and Cottrell, J.S. 1999. Probability-based protein identification by searching sequence databases using mass spectrometry data. Electrophoresis. 20:3551-3567. 